\title{
UNIVERSAL ABELIAN COVERS OF CERTAIN SURFACE SINGULARITIES
}

\author{
TOMOHIRO OKUMA
}

\begin{abstract}
Every normal complex surface singularity with $\mathbb{Q}$-homology sphere link has a universal abelian cover. It has been conjectured by Neumann and Wahl that the universal abelian cover of a rational or minimally elliptic singularity is a complete intersection singularity defined by a system of "splice diagram equations". In this paper we introduce a Neumann-Wahl system, which is an analogue of the system of splice diagram equations, and prove the following.

If $(X, o)$ is a rational or minimally elliptic singularity, then its universal abelian cover $(Y, o)$ is an equisingular deformation of an isolated complete intersection singularity $\left(Y_{0}, o\right)$ defined by a Neumann-Wahl system. Furthermore, if $G$ denotes the Galois group of the covering $Y \rightarrow X$, then $G$ also acts on $Y_{0}$ and $X$ is an equisingular deformation of the quotient $Y_{0} / G$.
\end{abstract}

\section{INTRODUCTION}

Let $(X, o)$ be a normal complex surface singularity germ. Let $\Gamma$ and $\Sigma$ denote the resolution graph and the link of $(X, o)$, respectively. We assume that $X$ is homeomorphic to the cone over $\Sigma$. It is known that the resolution graph and the link of the singularity determine each other $([6])$. Assume that the link $\Sigma$ is a $\mathbb{Q}$-homology sphere, or equivalently, that the exceptional set of a good resolution is a tree of rational curves. Then $H_{1}(\Sigma, \mathbb{Z})$ is finite. A morphism $(Y, o) \rightarrow(X, o)$ of germs of normal surface singularities is called a universal abelian covering if it induces an unramified Galois covering $Y \backslash\{o\} \rightarrow X \backslash\{o\}$ with covering transformation group $H_{1}(\Sigma, \mathbb{Z})$. By our assumption, the universal abelian covering $(Y, o) \rightarrow(X, o)$ must exist; in fact, the link of $Y$ is the universal abelian cover of $\Sigma$ in the topological sense. We are interested in the analytic properties of $Y$ and a way to construct $Y$ explicitly.

In the case that $(X, o)$ is quasihomogeneous, Neumann [7] proved that the universal abelian cover $(Y, o)$ is a Brieskorn-Pham complete intersection, by writing down the explicit equations from the data of $\Sigma$ (it is known that $\Gamma$ is star-shaped in this case). Neumann and Wahl generalized Brieskorn-Pham complete intersections and the way to construct them, and obtained numerous interesting results; see [8], [9], [10], [11]. They introduced the splice diagram equations (or forms) associated with a weighted tree called a splice diagram satisfying the "semigroup condition". From an arbitrary resolution graph corresponding to a $\mathbb{Q}$-homology sphere link,

2000 Mathematics Subject Classification. Primary 32S25; Secondary 14B05, 14J17.

Key words and phrases. abelian covering, rational surface singularity, minimally elliptic singularity, complete intersection singularity.

This research was partially supported by the Grant-in-Aid for Young Scientists (B), The Ministry of Education, Culture, Sports, Science and Technology, Japan. 
we can construct a splice diagram. Let $\widetilde{Y}$ denote the singularity defined by the splice diagram equations obtained from $\Gamma$. They proved that $\widetilde{Y}$ is an isolated complete intersection surface singularity, and that (under "congruence condition") if the equations are chosen so that the discriminant group $G\left(\cong H_{1}(\Sigma, \mathbb{Z})\right)$ for $\Gamma$ naturally acts on $\tilde{Y}$, then the quotient $\widetilde{Y} / G$ is a normal surface singularity (it is called a splice-quotient singularity) with resolution graph $\Gamma$, and the quotient morphism is the universal abelian covering. Neumann and Wahl conjectured that rational singularities and minimally elliptic singularities with $\mathbb{Q}$-homology sphere links are splice-quotient singularities. However, it is not known whether the splice diagrams obtained from the resolution graphs of those singularities satisfy the semigroup condition.

In this paper we prove that the universal abelian cover of a rational or minimally elliptic singularity is a complete intersection singularity defined by certain special functions.

Let $\pi: M \rightarrow X$ be a good resolution with the exceptional set $A$. Under a topological condition (Condition 3.3), we can associate a collection of certain special polynomials and a system of weights with each node of $A$. These polynomials are quasihomogeneous with respect to the weights. We call the union of those collections over all nodes a Neumann-Wahl system, which is an analogue of the system of splice diagram equations. Though the definition of a Neumann-Wahl system is very similar to that of splice diagram equations, they are not the same (see Remark 3.9). We suspect that a Neumann-Wahl system is a special type of system of splice diagram forms. Our first result is the following (see Theorem 4.3).

Theorem 1.1. Let $(V, o)$ be a singularity defined by a Neumann-Wahl system. Then $(V, o)$ is an isolated complete intersection surface singularity. A singularity defined by functions obtained by adding "higher terms" to the Neumann-Wahl system is an equisingular deformation of $(V, o)$.

We call a singularity defined by a Neumann-Wahl system a Neumann-Wahl complete intersection. If in addition a certain analytic condition (Condition 5.2) and a topological condition (Condition 3.4), which is stronger than Condition 3.3, are satisfied, then the universal abelian cover $Y$ is an equisingular deformation of a Neumann-Wahl complete intersection (Theorem 5.10). The equations of $Y$ are constructed on the resolution space $M$. Our equations for the deformation are automatically equivalent with respect to a natural action of the discriminant group $G$, and the action is free on nonsingular locus.

An important point is that Condition 3.4 and 5.2 are satisfied in case $(X, o)$ is a rational or minimally elliptic singularity (it can be easily verified!). Thus we have the following (see Theorem 5.1 and Proposition 5.14).

Theorem 1.2. If $(X, o)$ is a rational or minimally elliptic singularity, then its universal abelian cover $(Y, o)$ is an equisingular deformation of a Neumann-Wahl complete intersection singularity $\left(Y_{0}, o\right)$. Moreover the $(X, o)$ is an equisingular deformation of $\left(Y_{0} / G, o\right)$.

This paper is organized as follows. In Section 2, we briefly review fundamental results on the universal abelian covers of normal surface singularities in [12]. We recall there that $\mathcal{O}_{Y, o}$ is isomorphic to an $\mathcal{O}_{X, o}$-algebra $\mathcal{A}:=\bigoplus_{b \in \mathcal{B}} H^{0}\left(-L^{(b)}\right)$, where $\mathcal{B}$ is a group isomorphic to $G$ and $L^{(b)}$ are suitable divisors on $M$. In Section 3, we first define monomial cycles. The semigroup of monomial cycles is naturally 
isomorphic to that of monomials; the variables are associated with the ends of $A$. We show that Condition 3.4 implies Condition 3.3, and is satisfied for rational or minimally elliptic singularities. Then we introduce the Neumann-Wahl systems and the weights. In Section 4, we prove a slight generalization of Theorem 1.1; there we consider a system of polynomials obtained by substituting some power of the variables into the variables of a Neumann-Wahl system. We will apply some ideas in $[11, \S 2]$ for the proof. In the last section, we prove Theorem 1.2. We construct the equations by looking at certain relations of sections of $H^{0}\left(-L^{(b)}\right)$ 's. We can take a good basis of the algebra $\mathcal{A}$ by Condition 5.2, and can explicitly obtain the relations by Condition 3.4.

\section{Preliminaries}

In this section, we recall some fundamental results on the universal abelian covers of surface singularities; see [12] for details.

Let $(X, o)$ be a normal complex surface singularity germ. We assume that the link $\Sigma$ of $(X, o)$ is a $\mathbb{Q}$-homology sphere and that $X$ is homeomorphic to a cone over $\Sigma$. Then $X$ has a unique universal abelian cover. Let $\pi: M \rightarrow X$ be a resolution of the singularity, and let $A=\bigcup_{i} A_{i}$ be the decomposition of the exceptional set $A=\pi^{-1}(o)$ into irreducible components. Assume that $\pi$ is a good resolution, i.e., $A$ is a divisor having only simple normal crossings. Then the condition that $\Sigma$ is a $\mathbb{Q}$-homology sphere is equivalent to that $H^{1}\left(\mathcal{O}_{A}\right)=0$, i.e., $A$ is a tree of nonsingular rational curves. We call a divisor supported in $A$ a cycle. Let $A_{\mathbb{Z}}$ denote the group of cycles. An element of $A_{\mathbb{Q}}:=A_{\mathbb{Z}} \otimes \mathbb{Q}$ is called a $\mathbb{Q}$-cycle. Let $\bar{A}_{i} \in A_{\mathbb{Q}}$ denote the dual cycle of $A_{i}$, i.e., the $\mathbb{Q}$-cycle satisfying $\bar{A}_{i} \cdot A_{j}=-\delta_{i j}$, where $\delta_{i j}$ denotes the Kronecker delta. We denote by $\bar{A}_{\mathbb{Z}}$ the subgroup of $A_{\mathbb{Q}}$ generated by $\bar{A}_{i}$ 's. Recall that the first homology group $H_{1}(\Sigma, \mathbb{Z})$, the Galois group of the universal abelian covering of $X$, is isomorphic to the group $\bar{A}_{\mathbb{Z}} / A_{\mathbb{Z}}$ called the discriminant group. The order of the group is $\left|\operatorname{det}\left(A_{i} \cdot A_{j}\right)\right|$. Let $D$ be a $\mathbb{Q}$-divisor on $M$. We denote by $\nu(D)$ the $\mathbb{Q}$-cycle satisfying $(\nu(D)-D) \cdot A_{i}=0$ for all $A_{i}$. We say that $D$ is $\pi$-anti-nef if $-D$ is $\pi$-nef, i.e., $D \cdot A_{i} \leq 0$ for all $A_{i}$. Let $\mathcal{F}(D)$ denote the set of $\pi$-anti-nef $\mathbb{Q}$-divisors $F$ satisfying $F-D \in A_{\mathbb{Z}}$. Note that $\mathcal{F}(D)$ has the minimum with respect to " $\geq$ ".

We take effective $\mathbb{Q}$-cycles $E_{1}, \ldots, E_{s}$ such that if $\mathcal{E}_{i}$ denotes the cyclic subgroup of $\bar{A}_{\mathbb{Z}} / A_{\mathbb{Z}}$ generated by $E_{i}$, then $\bar{A}_{\mathbb{Z}} / A_{\mathbb{Z}}=\mathcal{E}_{1} \oplus \cdots \oplus \mathcal{E}_{s}$. Let $r_{i}$ be the order of $\mathcal{E}_{i}$. Then for $1 \leq i \leq s$ there exists a divisor $L_{i}$ and a function $f_{i}$ on a suitable neighborhood of $A$ such that $r_{i} L_{i}-r_{i} E_{i}=\operatorname{div}\left(f_{i}\right)$. For any $b=\left(b_{1}, \ldots, b_{s}\right) \in \mathbb{Z}^{s}$, we define a divisor $L^{(b)}$ by

$$
L^{(b)}=\sum_{j=1}^{s} b_{j} L_{j}-\left[\sum_{j=1}^{s} b_{j} E_{j}\right] .
$$

Let $\bar{b}_{i}$ denote the smallest nonnegative integer such that $r_{i} \mid b_{i}-\bar{b}_{i}$. Let $\bar{b}=$ $\left(\bar{b}_{1}, \ldots, \bar{b}_{s}\right)$ and $\mathcal{B}=\left\{\bar{b} \mid b \in \mathbb{Z}^{s}\right\}$; the set $\mathcal{B}$ is identified with the discriminant group $\bar{A}_{\mathbb{Z}} / A_{\mathbb{Z}}$. We define a sheaf $\overline{\mathcal{A}}$ of $\mathcal{O}_{M}$-modules by

$$
\overline{\mathcal{A}}=\bigoplus_{b \in \mathcal{B}} \mathcal{O}_{M}\left(-L^{(b)}\right)
$$


The $\mathcal{O}_{M}$-algebra structure of $\overline{\mathcal{A}}$ is given by the composite

$$
\mathcal{O}_{M}\left(-L^{(b)}\right) \otimes \mathcal{O}_{M}\left(-L^{\left(b^{\prime}\right)}\right) \rightarrow \mathcal{O}_{M}\left(-L^{(b)}-L^{\left(b^{\prime}\right)}\right) \subset \mathcal{O}_{M}\left(-L^{\left(b+b^{\prime}\right)}\right)
$$

and the isomorphism

$$
\mathcal{O}_{M}\left(-L^{\left(b+b^{\prime}\right)}\right) \rightarrow \mathcal{O}_{M}\left(-L^{\left(\overline{b+b^{\prime}}\right)}\right)
$$

given by multiplying by $\prod_{b_{i}+b_{i}^{\prime} \neq \overline{b_{i}+b_{i}^{\prime}}} f_{i}^{-1}$. Then the natural projection

$$
Y:=\operatorname{Specan}_{X} \pi_{*} \overline{\mathcal{A}} \rightarrow X
$$

is the universal abelian covering (see [12, Theorem 3.4]). The local ring $\mathcal{O}_{Y, o}$ of the singularity $(Y, o)$ is isomorphic to

$$
\mathcal{A}:=\left(\pi_{*} \overline{\mathcal{A}}\right)_{o}=\bigoplus_{b \in \mathcal{B}} H^{0}\left(-L^{(b)}\right),
$$

where $H^{0}\left(-L^{(b)}\right)=\operatorname{dir} \lim _{U} H^{0}\left(U, \mathcal{O}_{M}\left(-L^{(b)}\right)\right), U$ varies over all open neighborhoods of $A$. We write $\mathcal{A}_{b}=H^{0}\left(-L^{(b)}\right)$.

Let $h \in \mathcal{A}_{b}$. If $\operatorname{div}(h)-L^{(b)}-D$ has no component of $A$ for some cycle $D \in A_{\mathbb{Z}}$, then we write $(h)_{A}=\nu\left(L^{(b)}\right)+D$.

Lemma 2.1. Let $\sigma_{i} \in \mathcal{A}_{b^{i}}, i=1,2$, and let $\sigma_{1} \cdot \sigma_{2} \in \mathcal{A}_{\overline{b^{1}+b^{2}}}$ be the product of $\sigma_{1}$ and $\sigma_{2}$ in the algebra $\mathcal{A}$. Suppose that a divisor $L \in \mathcal{F}\left(L^{\left(\overline{b^{1}+b^{2}}\right)}\right)$ satisfies $\nu(L)=\left(\sigma_{1}\right)_{A}+\left(\sigma_{2}\right)_{A}$. Then $\sigma_{1} \cdot \sigma_{2}$ is a section of $H^{0}(-L)$ and $\left(\sigma_{1} \cdot \sigma_{2}\right)_{A}=\nu(L)$.

Proof. It follows from the definition of the algebra structure of $\mathcal{A}$.

Let $D$ be a reduced and connected cycle. A component $A_{i}$ of $D$ is called an end of $D$ if $\left(D-A_{i}\right) \cdot A_{i} \leq 1$. We denote by $\mathcal{E}(D)$ the set of ends of $D$.

Assume that $\mathcal{E}(A)=\left\{A_{1}, \ldots, A_{m}\right\}$. For any $1 \leq i \leq m$, there uniquely exists a divisor $L^{i}$ such that $\nu\left(L^{i}\right)=\bar{A}_{i}$ and $L^{i} \in \mathcal{F}\left(L^{(b)}\right)$ for some $b \in \mathcal{B}$. If $(X, o)$ is rational, then there exists $y_{i} \in H^{0}\left(-L^{i}\right)$ such that $\left(y_{i}\right)_{A}=\nu\left(L^{i}\right)$ (cf. Lemma 5.3). The next theorem follows from [12, Theorem 7.5] and its proof.

Theorem 2.2. Assume that $(X, o)$ is rational. Let $y_{i} \in H^{0}\left(-L^{i}\right), 1 \leq i \leq m$, be as above. Let $S=\mathbb{C}\left\{x_{1}, \ldots, x_{m}\right\}$ be the convergent power series ring. We define a homomorphism $\psi: S \rightarrow \mathcal{A}=\mathcal{O}_{Y, o}$ of $\mathbb{C}$-algebras by $\psi\left(x_{i}\right)=y_{i}$. Then $\psi$ is surjective.

In the last section, we give a proof of Theorem 2.2, which is different from that in [12]. In fact it is shown that the assertion also holds true for minimally elliptic singularities.

\section{NeUmann-Wahl Systems}

In this section we will introduce a Neumann-Wahl system associated with the exceptional set $A$. It is a set of certain polynomials, and an analogue of the system of splice diagram equations in Neumann and Wahl's work ([8], [9], [11]).

We use the notation of the preceding section, and keep the assumption that $H^{1}\left(\mathcal{O}_{A}\right)=0$. First assume that the set $\mathcal{E}(A)$ consists of the components $A_{1}, \ldots, A_{m}$. Let $\mathbb{C}\left[x_{1}, \ldots, x_{m}\right]$ be the polynomial ring. Theorem 2.2 suggests us the following 
Definition 3.1. Let $D=\sum a_{i} \bar{A}_{i} \in A_{\mathbb{Q}}, a_{i} \geq 0$. If $a_{i}=0$ for all $A_{i} \notin \mathcal{E}(A)$, then we call $D$ a $\mathbb{Q}$-monomial cycle; if in addition $a_{i} \in \mathbb{Z}$ for all $i$, we call $D$ a monomial cycle. For any monomial cycle $D=\sum_{i=1}^{m} a_{i} \bar{A}_{i}$, we associate a monomial

$$
x(D):=\prod_{i=1}^{m} x_{i}^{a_{i}} \in \mathbb{C}\left[x_{1}, \ldots, x_{m}\right] .
$$

The $x$ induces an isomorphism between the semigroup of monomial cycles and that of monomials of $x_{1}, \ldots, x_{m}$. Formally, we may also consider the $\mathbb{Q}$-monomial $x(D)$ for a $\mathbb{Q}$-monomial cycle $D$.

Definition 3.2. For any $F=\sum a_{k} A_{k} \in A_{\mathbb{Q}}$, we write $m_{A_{k}}(F)=a_{k}$. For any component $A_{j}$, we define the $A_{j}$-weight of $x_{i}$ to be $m_{A_{j}}\left(\bar{A}_{i}\right)$. Then the $A_{j}$-degree of a monomial $x(D)$ is defined to be $m_{A_{j}}(D)$.

A connected component of $A-A_{i}$ is called a branch of $A_{i}$. A component $A_{i}$ is called a node if $\left(A-A_{i}\right) \cdot A_{i} \geq 3$. We consider the following conditions concerning the weighted dual graph of $A$.

Condition 3.3. For any branch $C$ of any node $A_{i}$, there exists a monomial cycle $D$ such that $D-\bar{A}_{i}$ is an effective integral cycle supported on $C$; in this case, we say that $D$ (or monomial $x(D)$ ) belongs to the branch $C$.

Note that in general there may exist more than one monomials belonging to a branch.

Condition 3.4. $A$ is star-shaped, or for any branch $C$ of any component $A_{i} \notin \mathcal{E}(A)$, the fundamental cycle $Z_{C}$ supported on $C$ satisfies $Z_{C} \cdot A_{i}=1$.

Lemma 3.5. Condition 3.4 implies Condition 3.3, and is satisfied in the following cases:

(1) $(X, o)$ is a rational singularity;

(2) $(X, o)$ is a minimally elliptic singularity, and the minimally elliptic cycle is supported on $A$ (this condition is satisfied on the minimal good resolution).

Proof. Assume that the condition (1) or (2) is satisfied. From basic results on the computation sequences for the fundamental cycle ([3], [4]), we obtain Condition 3.4. Suppose that $C_{1}$ is a branch of a node $A_{i_{1}}$. Then $D_{1}:=\bar{A}_{i_{1}}+Z_{C_{1}}$ is $\pi$-anti-nef and $D_{1} \cdot A_{j}=0$ for every $A_{j} \leq A-C_{1}$. If $D_{1} \cdot A_{i_{2}}<0$ for $A_{i_{2}} \notin \mathcal{E}(A)$, then take a branch $C_{2}$ of $A_{i_{2}}$, not containing $A_{i_{1}}$, and put $D_{2}:=D_{1}+Z_{C_{2}}$. In this manner we obtain a finite sequence $\left\{D_{1}, \ldots, D_{n}\right\}$ of $\pi$-anti-nef cycles, which ends with a monomial cycle belonging to $C_{1}$. Thus Condition 3.3 is satisfied. These arguments also show that Condition 3.3 holds in case $A$ is star-shaped.

Definition 3.6. Assume that Condition 3.3 is satisfied, and that $A_{1}, \ldots, A_{s}$ are all of the nodes of $A$. Let $C_{1}, \ldots, C_{p}$ be the branches of $A_{1}$.

(1) A monomial $x(D)$ is called an admissible monomial at the node $A_{1}$ if it belongs to one of the branches of $A_{1}$. A set of monomials $\left\{x\left(D_{1}\right), \ldots, x\left(D_{p}\right)\right\}$ is called a complete system of admissible monomials at $A_{1}$ if $D_{i}$ belongs to $C_{i}$ for $i=1, \ldots, p$.

(2) Let $\left\{m_{1}, \ldots, m_{p}\right\}$ be any complete system of admissible monomials at $A_{1}$. Let $F=\left(c_{i j}\right), c_{i j} \in \mathbb{C}$, be a $((p-2) \times p)$-matrix such that every maximal minor of 
it has rank $p-2$. We define polynomials $f_{1}, \ldots, f_{p-2}$ by

$$
\left(\begin{array}{c}
f_{1} \\
\vdots \\
f_{p-2}
\end{array}\right)=F\left(\begin{array}{c}
m_{1} \\
\vdots \\
m_{p}
\end{array}\right) .
$$

We call each $f_{i}$ an admissible form at $A_{1}$ and the set $\left\{f_{1}, \ldots, f_{p-2}\right\}$ a NeumannWahl system at $A_{1}$.

(3) Let $\mathcal{F}_{i}$ denote a Neumann-Wahl system at a node $A_{i}$. Then we call the set $\bigcup_{i=1}^{s} \mathcal{F}_{i}$ a Neumann-Wahl system associated with $A$; it is an empty set in case $A$ has no nodes.

Remark 3.7. The matrix $F$ above can be reduced to the following matrix by row operations:

$$
\left(\begin{array}{cccccc}
1 & 0 & \ldots & 0 & a_{1} & b_{1} \\
0 & 1 & \ldots & 0 & a_{2} & b_{2} \\
\vdots & \vdots & \ddots & \vdots & \vdots & \vdots \\
0 & 0 & \ldots & 1 & a_{p-2} & b_{p-2}
\end{array}\right),
$$

where $a_{i} b_{j}-a_{j} b_{i} \neq 0$ for $i \neq j$, and all $a_{i}$ and $b_{i}$ are nonzero.

The admissible forms at a node $A_{i}$ are quasihomogeneous polynomials with respect to the $A_{i}$-weight. The following lemma is needed in the next section.

Lemma 3.8. Let $D$ be a $\pi$-anti-nef $\mathbb{Q}$-cycle such that $m_{A_{i}}(D)>m_{A_{i}}\left(\bar{A}_{i}\right)$ for some $i$. Then for any component $A_{j}$, we have $m_{A_{j}}(D)>m_{A_{j}}\left(\bar{A}_{i}\right)$.

Proof. First we will show that $D \geq \bar{A}_{i}$. There exist effective $\mathbb{Q}$-cycles $D_{1}$ and $D_{2}$ such that $D-\bar{A}_{i}=D_{1}-D_{2}$ and that $D_{1}$ and $D_{2}$ have no common components. If $D_{2}>0$, then

$$
m_{A_{i}}\left(D_{2}\right)=-\bar{A}_{i} \cdot D_{2}=-D \cdot D_{2}+D_{1} \cdot D_{2}-D_{2} \cdot D_{2}>0 .
$$

It contradicts the assumption of the lemma. Hence $D-\bar{A}_{i} \geq 0$.

Let $a=m_{A_{i}}\left(D-\bar{A}_{i}\right)$ and $C$ a branch of $A_{i}$. If a component $A_{k}$ of $C$ intersects $A_{i}$, then $\left(\bar{A}_{i}+a A_{i}\right) \cdot A_{k}=a>0$. Since $D$ is $\pi$-anti-nef, there exists a $\mathbb{Q}$-cycle $C^{\prime}$ supported on $C$ such that $C^{\prime} \cdot A_{l} \leq 0$ for all $A_{l} \leq C$ and that $D \geq \bar{A}_{i}+a A_{i}+C^{\prime}$.

Remark 3.9. The definition of a Neumann-Wahl system is very similar to that of a system of splicing diagram equations in the Neumann and Wahl's work. However those are not the same. Let us consider a weighted graph $\Gamma$ represented as in Figure 1; the vertex has weight -4 and other vertices $\bullet$ have weight -2 . The graph $\Gamma$

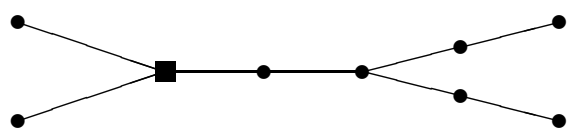

FiguRE 1.

is realized as the resolution graph of an elliptic singularity with $\mathbb{Q}$-homology sphere link. The splice diagram $\Delta$ associated with $\Gamma$ is represented as in Figure 2. We see that $\Delta$ satisfies semigroup condition. However, $\Gamma$ does not satisfy Condition 3.3. Therefore we cannot define Neumann-Wahl systems in this case, though the splice diagram equations are defined. That might indicate a Neumann-Wahl system is a special type of system of splice diagram equations. 


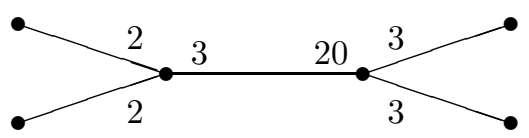

FiguRE 2.

\section{VARIETIES DEFINED By NeUmanN-WAhl Systems}

In this section we prove that any Neumann-Wahl system defines a complete intersection surface with an isolated singularity at the origin; so we will call such a singularity a Neumann-Wahl complete intersection singularity. In fact, we will prove the assertion for a slight generalization of a Neumann-Wahl system. We note that some of methods in this section are discussed in [11].

We use the notation of the preceding section. Assume that Condition 3.3 is satisfied and that $A$ has at least one node. As in the preceding section, we associate ends of $A$ with the variables $x_{1}, \ldots, x_{m}$, where $m=\# \mathcal{E}(A)$. Suppose that $A_{1}, \ldots, A_{s}$ are all of the nodes of $A$. Let $d_{i}$ denote the number of branches of a node $A_{i}$. Let $\mathcal{M}_{i}=\left\{m_{i 1}, \ldots, m_{i d_{i}}\right\}$ denote a complete system of admissible monomials and $\mathcal{F}_{i}=\left\{f_{i 1}, \ldots, f_{i d_{i}-2}\right\}$ a Neumann-Wahl system at a node $A_{i}$, where each $f_{i j}$ is a linear form of monomials of $\mathcal{M}_{i}$. By counting the numbers of the ends, the nodes and the edges around the nodes of the dual graph of $A$, we see that

$$
\sum_{i=1}^{s}\left(d_{i}-2\right)=m-2 .
$$

Let $C_{1}, \ldots, C_{d_{1}}$ denote the branches of $A_{1}$. Without loss of generality, we may assume the following.

(1) For $1 \leq j \leq d_{1}-1, C_{j}$ is a chain of curves; in this case, $A_{1}$ is an end of the minimal reduced connected cycle containing all nodes of $A$.

(2) For $1 \leq j \leq d_{1}-1$, the variable $x_{j}$ corresponds to the end of $C_{j}$.

(3) For $2 \leq i \leq s$, the admissible monomial $m_{i d_{i}}$ belongs to the branch of $A_{i}$ containing $A_{1}$.

(4) For $1 \leq i \leq s$, the admissible forms of $\mathcal{F}_{i}$ are given by the matrix as in Remark 3.7; we write

$$
\begin{aligned}
f_{i j} & =m_{i j}+a_{i j} m_{i d_{i}-1}+b_{i j} m_{i d_{i}}, & & 1 \leq i \leq s, 1 \leq j \leq d_{i}-2, \\
m_{1 j} & =x_{j}^{\alpha_{j}}, & & 1 \leq j \leq d_{1}-1 .
\end{aligned}
$$

Now we slightly modify the admissible forms. This modification is needed for the induction step of the proof of the main theorem. Let $\mathbb{N}$ denote the set of positive integers. A vector $v \in \mathbb{N}^{m}$ is said to be primitive if $v$ cannot be written as $v=c v^{\prime}$ with $v^{\prime} \in \mathbb{N}^{m}$ and $c \in \mathbb{N}, c>1$. Fix an arbitrary vector $\delta=\left(\delta_{1}, \ldots, \delta_{m}\right) \in \mathbb{N}^{m}$. For each node $A_{i}$, let $e_{i}$ denote the positive integer such that

$$
\mathbf{w}_{i}:=\left(A_{i}-\operatorname{deg}\left(x_{1}\right) \cdot e_{i} / \delta_{1}, \ldots, A_{i}-\operatorname{deg}\left(x_{m}\right) \cdot e_{i} / \delta_{m}\right) \in \mathbb{N}^{m}
$$

is primitive. Let $S=\mathbb{C}\left\{x_{1}, \ldots, x_{m}\right\}$ be the convergent power series ring.

Definition 4.1. Let $\mathbf{w}=\left(w_{1}, \ldots, w_{m}\right) \in \mathbb{N}^{m}$. We define the $\mathbf{w}$-degree of a $\mathbb{Q}$ monomial $m=\prod_{k=1}^{m} x_{k}^{a_{k}}$ to be $\mathbf{w}-\operatorname{deg}(m)=\sum a_{k} w_{k}$. Let $f=\sum_{k \geq 1} f_{k} \in S$, where $f_{1} \neq 0$ and each $f_{k}$ is a quasihomogeneous polynomial with respect to $\mathbf{w}$ such that $\mathbf{w}-\operatorname{deg}\left(f_{k}\right)<\mathbf{w}-\operatorname{deg}\left(f_{k+1}\right)$. We call $f_{1}$ the leading form of $f$, and denote it by 
$\operatorname{LF}_{\mathbf{w}}(f)$. Then $f-\mathrm{LF}_{\mathbf{w}}(f)$ is called the higher term of $f$. We define $\mathbf{w}$-order of $f$ to be $\mathbf{w}-\operatorname{ord}(f)=\mathbf{w}-\operatorname{deg}\left(\operatorname{LF}_{\mathbf{w}}(f)\right)$. We set $\mathbf{w}-\operatorname{ord}(0)=\infty$.

We write $\mathbf{x}_{k}=x_{k}^{\delta_{k}}$ and $\mathbf{f}=f\left(\mathbf{x}_{1}, \ldots, \mathbf{x}_{m}\right)$ for $f=f\left(x_{1}, \ldots, x_{m}\right) \in S$. We call $\mathbf{f}$ the $\delta$-lifting of $f$. Any monomial can be thought as the $\delta$-lifting of a $\mathbb{Q}$-monomial. A polynomial $f$ is quasihomogeneous with respect to $A_{i}$-weight if and only if so is f with respect to the weight $\mathbf{w}_{i}$; in fact, for a $\mathbb{Q}$-monomial $m$, we have

$$
\mathbf{w}_{i}-\operatorname{deg}(\mathbf{m})=A_{i}-\operatorname{deg}(m) \cdot e_{i} .
$$

For each $\mathbf{f}_{i j}$, we take a convergent power series $f_{i j}^{+} \in S$ satisfying

$$
\mathbf{w}_{i}-\operatorname{deg}\left(\mathbf{f}_{i j}\right)<\mathbf{w}_{i} \text {-ord }\left(f_{i j}^{+}\right) .
$$

For each $t \in \mathbb{C}$, we set

$$
\mathcal{F}_{t}=\left\{\mathbf{f}_{i j}+t f_{i j}^{+} \mid 1 \leq i \leq s, 1 \leq j \leq d_{i}-2\right\} .
$$

We note that if $m_{i j}=x\left(D_{i j}\right)$, then for $i \geq 2$ and $1 \leq j \leq d_{i}-1$,

$$
m_{A_{1}}\left(D_{i d_{i}}\right)>m_{A_{1}}\left(D_{i j}\right)=m_{A_{1}}\left(\bar{A}_{i}\right) \text {. }
$$

If the $\delta$-lifting of a $\mathbb{Q}$-monomial $x(D)$ is contained in $f_{i j}^{+}$, then $m_{A_{i}}(D)>m_{A_{i}}\left(\bar{A}_{i}\right)$. By Lemma 3.8, $m_{A_{1}}(D)>m_{A_{1}}\left(\bar{A}_{i}\right)$. Thus we obtain the following:

$$
\begin{aligned}
& \mathrm{LF}_{\mathbf{w}_{1}}\left(\mathbf{f}_{1 j}+t f_{1 j}^{+}\right)=\mathbf{f}_{1 j}, \\
& \mathrm{LF}_{\mathbf{w}_{1}}\left(\mathbf{f}_{i j}+t f_{i j}^{+}\right)=\mathbf{m}_{i j}+a_{i j} \mathbf{m}_{i d_{i}-1}, \quad \text { for } i \geq 2 .
\end{aligned}
$$

Therefore the set

$$
\mathrm{LF}_{\mathbf{w}_{1}} \mathcal{F}:=\left\{\mathrm{LF}_{\mathbf{w}_{1}}(f) \mid f \in \mathcal{F}_{t}\right\}
$$

is independent of $t \in \mathbb{C}$; it can be shown that $\mathrm{LF}_{\mathbf{w}_{i}} \mathcal{F}$ is also independent of $t$ for $2 \leq i \leq s$.

Definition 4.2 (Wahl [15], cf. [5, V]). Let $\omega: \widetilde{X} \rightarrow T$ be a deformation of a normal surface singularity $\widetilde{X}_{o}=\omega^{-1}(o), o \in T$. Suppose that each fiber $\widetilde{X}_{t}$ has only one singular point and that there exists a simultaneous resolution $\bar{\omega}: \widetilde{M} \rightarrow \widetilde{X}$ with the exceptional set $\widetilde{A}$. If the restriction $\left.(\omega \circ \bar{\omega})\right|_{\widetilde{A}}$ is a locally trivial deformation of the exceptional divisor of $\widetilde{M}_{o}$, then we call $\omega \circ \bar{\omega}$ (resp. $\omega$ ) an equisingular deformation. $\bar{\omega}$ is called a weak simultaneous resolution of $\omega$.

For a subset $B$ of any commutative ring, let $I(B)$ denote the ideal generated by the elements of $B$.

Let $\left(V_{t}, o\right) \subset\left(\mathbb{C}^{m}, o\right)$ denote the singularity defined by the ideal $I\left(\mathcal{F}_{t}\right) \subset S$. The main result of this section is the following.

Theorem 4.3 (cf. [11, Theorem 2.6]). The singularity $\left(V_{t}, o\right)$ is an isolated complete intersection surface singularity for each $t \in \mathbb{C}$. Furthermore, the family $\left\{V_{t} \mid t \in \mathbb{C}\right\}$ is an equisingular deformation.

First we show that every $V_{t}$ is a complete intersection.

Lemma 4.4 (cf. [11, Theorem 3.1]). For any variable $x_{k}$, let $A_{i_{k}}$ denote the node nearest to the end corresponding to $x_{k}$. Let $C \subset \mathbb{C}^{m}$ be the affine variety defined by the ideal $I\left(\mathrm{LF}_{\mathbf{w}_{i_{k}}} \mathcal{F} \cup\left\{x_{k}\right\}\right)$.

(1) If $x_{j}=0(j \neq k)$ at $p \in C$, then $p$ is the origin.

(2) $C$ is a complete intersection curve and smooth except for the origin. 
Proof. Without loss of generality, we may assume that $k=1$. Let

$$
c_{j}= \begin{cases}b_{11} / a_{11} & \text { if } j=d_{1}-1, \\ b_{1 j}-a_{1 j} b_{11} / a_{11} & \text { if } 2 \leq j \leq d_{1}-2 .\end{cases}
$$

Then $c_{j} \neq 0$ and $C$ is a subvariety of the affine space $\mathbb{C}^{m-1}$ with coordinates $x_{2}, \ldots, x_{m}$ defined by the equations

$$
\begin{aligned}
\mathbf{x}_{j}^{\alpha_{j}}+c_{j} \mathbf{m}_{1 d_{1}}=0, & 2 \leq j \leq d_{1}-1, \\
\mathbf{m}_{i j}+a_{i j} \mathbf{m}_{i d_{i}-1}=0, & 2 \leq i \leq s, 1 \leq j \leq d_{i}-2 .
\end{aligned}
$$

If a monomial appearing in (4.2) vanishes at $p \in C$, then so does every monomial in the equations at the same node. On the other hand, for each $2 \leq i \leq m$, some power of $x_{i}$ appears in (4.2) because each end of $A$ is a unique end of a branch of the nearest node. Thus if a variable $x_{i}(i \geq 2)$ vanishes on $C$, then so do all monomials appearing in (4.2), since $A$ is a connected tree of curves. Hence we obtain (1).

Since $\# \mathrm{LF}_{\mathbf{w}_{1}} \mathcal{F}=m-2$, it follows from (1) that $\left\{x_{1}, x_{i}\right\} \cup \mathrm{LF}_{\mathbf{w}_{1}} \mathcal{F}$, where $i \neq 1$, is a regular sequence. Hence $C$ is one-dimensional and complete intersection. The argument above also shows that an ideal $I\left(\left\{x_{1}, x_{i}-1\right\} \cup L_{\mathbf{w}_{1}} \mathcal{F}\right)$ defines a nonsingular zero-dimensional variety. Since $C$ is defined by quasihomogeneous polynomials, $C$ is smooth except for the origin.

Corollary 4.5 (cf. [11, Corollary 3.4]). $V_{t}$ is a complete intersection surface singularity, and the support of $V_{t} \cap\left\{x_{j}=x_{k}=0\right\}, j \neq k$, is the origin.

Proof. By Lemma 4.4, $\mathrm{LF}_{\mathbf{w}_{i_{k}}} \mathcal{F} \cup\left\{x_{j}, x_{k}\right\}$ is a regular sequence. Hence so are $\mathcal{F}_{t}$ and $\mathcal{F}_{t} \cup\left\{x_{j}, x_{k}\right\}$.

Definition 4.6. We define the weighted dual graph of a normal surface singularity to be that of the exceptional set of the minimal good resolution of the singularity.

Let $(W, o)$ be a germ of a normal surface singularity and $W^{\prime} \rightarrow W$ the minimal resolution. The canonical cycle on $W^{\prime}$ is a $\mathbb{Q}$-cycle which is numerically equivalent to the canonical divisor $K_{W^{\prime}}$. The self-intersection number of the canonical cycle is an invariant of the singularity and determined by the weighted dual graph; we denote it by $K^{2}(W)$. Let $\omega: \widetilde{X} \rightarrow T \subset \mathbb{C}$ be a deformation of surface singularities. The invariance of $K^{2}\left(\widetilde{X}_{t}\right)$ implies the existence of the simultaneous canonical model (or simultaneous RDP resolution) of $\omega$; first the Gorenstein case was proved by Laufer ([5, Theorem 4.3]), and the general case by Ishii ([2, Corollary 1.10]). By [1], the singularities of the simultaneous canonical model are simultaneously resolved after a suitable finite base change. Thus we obtain the following theorem by the arguments of $[5, \mathrm{VI}]$.

Theorem 4.7 (Laufer, Ishii). Let $\omega: \widetilde{X} \rightarrow T \subset \mathbb{C}$ be a deformation of a normal surface singularity. If the weighted dual graphs of $\widetilde{X}_{t}, t \in T$, are the same, then $\omega$ is an equisingular deformation, and it admits a simultaneous resolution such that each fiber is the minimal good resolution.

For a divisor $D$, we denote by $D_{\text {red }}$ the reduced divisor with $\operatorname{Supp}\left(D_{\text {red }}\right)=$ $\operatorname{Supp}(D)$. For the induction step of the proof of the main theorem, we need the following. 
Lemma 4.8. Let $\omega: \widetilde{X} \rightarrow \mathbb{C}$ be an equisingular deformation of a germ of a normal surface singularity $\widetilde{X}_{0}=\omega^{-1}(0)$. Let $\widetilde{D}$ be a reduced divisor on $\widetilde{X}$, which contains the singular locus of $\widetilde{X}$. Suppose that $\left.\omega\right|_{\widetilde{D}}$ is a locally trivial deformation of a reduced divisor $\widetilde{D}_{0}:=\left.\widetilde{D}\right|_{\widetilde{X}_{0}}$ on $\widetilde{X}_{0}$. Then there exists a simultaneous resolution $\bar{\omega}: \widetilde{M} \rightarrow \widetilde{X}$ with the exceptional set $\widetilde{A}$ such that $\left.(\omega \circ \bar{\omega})\right|_{\left(\bar{\omega}^{*} \widetilde{D}\right)_{\text {red }}}$ is a locally trivial deformation (hence so is $\left.(\omega \circ \bar{\omega})\right|_{\tilde{A}}$ ).

Proof. There exists a weak simultaneous resolution $\bar{\omega}: \widetilde{M} \rightarrow \widetilde{X}$ with the exceptional set $\widetilde{A}$ such that each $\widetilde{A}_{t}:=\left.\widetilde{A}\right|_{\widetilde{M}_{t}}$ has only simple normal crossing and $\left.(\omega \circ \bar{\omega})\right|_{\widetilde{A}}$ is a locally trivial deformation. Let $\widetilde{F}$ denote the strict transform of $\widetilde{D}$ on $\widetilde{M}$. Let $\widetilde{A}^{1}$ (resp. $\widetilde{F}^{1}$ ) be a divisor on $\widetilde{M}$, which is the total space of the deformation of an irreducible component of $\widetilde{A}_{0}$ (resp. $\widetilde{F}_{0}:=\left.\widetilde{F}\right|_{\widetilde{M}_{0}}$ ). Then the intersection number $\widetilde{A}_{t}^{1} \cdot \widetilde{F}_{t}^{1}$ is constant. We may assume $\#\left(\widetilde{A}_{t}^{1} \cap \widetilde{F}_{t}^{1}\right) \leq 1$. If $\widetilde{A}_{t}^{1} \cdot \widetilde{F}_{t}^{1} \geq 2$, then take the blowing up of $\widetilde{M}$ along the curve $\widetilde{A}^{1} \cap \widetilde{F}^{1}$. By taking blowing ups successively in a similar way, we obtain a simultaneous resolution $\bar{\omega}^{\prime}: \widetilde{M}^{\prime} \rightarrow \widetilde{X}$ such that each divisor $\left(\left(\left.\bar{\omega}^{\prime}\right|_{\widetilde{M}_{t}^{\prime}}\right)^{*} \widetilde{D}_{t}\right)_{\text {red }}$ has only normal crossings and that the weighted dual graph of the divisor is independent of $t \in \mathbb{C}$.

Lemma 4.9. Let $\omega: \widetilde{X} \rightarrow \mathbb{C}$ be as in Lemma 4.8. Suppose that $\tilde{X}$ is embedded in an open subset of $\mathbb{C}^{n} \times \mathbb{C}$ such that the singular locus of $\widetilde{X}$ is $\{o\} \times \mathbb{C}$, and that $\omega$ is the composite of this embedding and the projection $\mathbb{C}^{n} \times \mathbb{C} \rightarrow \mathbb{C}$. Let $G$ be a finite subgroup of the unitary group $\mathrm{U}(n) \subset \mathrm{GL}\left(\mathbb{C}^{n}\right)$. Then $G$ acts on $\mathbb{C}^{n} \times \mathbb{C}$ by $g \cdot(z, t)=(g \cdot z, t), g \in G$. Assume the action induces an action on $\widetilde{X}$ which is free on $\widetilde{X} \backslash\{o\} \times \mathbb{C}$. Then the morphism $\mathcal{O}$ mega: $\widetilde{X} / G \rightarrow \mathbb{C}$ obtained from $\omega$ is an equisingular deformation of $\left(\widetilde{X}_{t} / G, o\right), t \in \mathbb{C}$.

Proof. Let $\mathbf{S}_{c} \subset \mathbb{C}^{n}, c>0$, denote the $(2 n-1)$-sphere of radius $c$. Let $t_{0} \in \mathbb{C}$ be an arbitrary point. Then there exist an open neighborhood $U$ of $t_{0}$ and a positive number $\epsilon \in \mathbb{R}$ such that $\Sigma_{t}:=\mathbf{S}_{\epsilon} \cap \widetilde{X}_{t} \subset \mathbb{C}^{n}$ is the link of $\widetilde{X}_{t}$ for every $t \in U$ and the family $\left\{\Sigma_{t} \mid t \in U\right\}$ is topologically trivial. By the assumption, $G$ acts on $\left\{\Sigma_{t} \mid t \in U\right\}$ freely. Thus we obtain a family $\left\{\Sigma_{t} / G \mid t \in U\right\}$ which is topologically trivial. Recall that the weighted dual graph of a surface singularity is determined by its link (Neumann [6]). By Theorem 4.7, we obtain the assertion.

We mention the weighted blowing up which is needed in the proof of the theorem. Let $\mathbf{w}=\left(w_{1}, \ldots, w_{m}\right) \in \mathbb{N}^{m}$ be a primitive vector, and let $\beta: Z \rightarrow \mathbb{C}^{m}$ be the weighted blowing up with respect to the weight $\mathbf{w}$. It is a projective morphism inducing an isomorphism $Z \backslash \beta^{-1}(o) \rightarrow \mathbb{C}^{m} \backslash\{o\}$, and $\beta^{-1}(o)=\mathbb{P}(\mathbf{w})$, the weighted projective space of type $\mathbf{w}$. The variety $Z$ is covered by affine varieties $Z_{1}, \ldots, Z_{m}$; each $Z_{i}$ is a quotient of $W_{i}=\mathbb{C}^{m}$ by a cyclic group $\mathcal{C}_{i}$ of order $w_{i}$ determined by the weight w. Let $\left\{x_{1}, \ldots, x_{m}\right\}$ and $\left\{z_{1}, \ldots, z_{m}\right\}$ be the coordinates of $\mathbb{C}^{m}$ and $W_{1}$, respectively. The action of the group $\mathcal{C}_{1}$ on $W_{1}$ is given by the diagonal matrix

$$
\operatorname{Diag}\left[e\left(-1 / w_{1}\right), e\left(w_{2} / w_{1}\right), \ldots, e\left(w_{m} / w_{1}\right)\right],
$$

where $e(q)=\exp (2 \pi \sqrt{-1} q)$. Since $\mathbf{w}$ is primitive, $\mathcal{C}_{1}$ is trivial or the fixed locus is a proper subvariety of the hyperplane $\left\{x_{1}=0\right\}$ which is the exceptional locus. The morphism $W_{1} \rightarrow \mathbb{C}^{m}$, which is the composite of the quotient morphism $W_{1} \rightarrow Z_{1}$ 
and $\beta: Z_{1} \rightarrow \mathbb{C}^{m}$, is given by

$$
x_{1}=z_{1}^{w_{1}}, \quad x_{i}=z_{1}^{w_{i}} z_{i} \quad(i=2, \ldots, m) .
$$

Proof of Theorem 4.3. We prove the theorem by induction on the number of nodes $s$ of $A$. We have to show the isolated singularity of each $V_{t}$ and the equisingularity of the family $\left\{V_{t} \mid t \in \mathbb{C}\right\}$.

First assume that $s=1$. Then $V_{0}$ is the so-called Brieskorn-Pham complete intersection singularity; it is known that $V_{0}$ has an isolated singularity (it is also easily checked by using the Jacobian criterion). We fix $t \in \mathbb{C}$. Since $\mathrm{LF}_{\mathbf{w}_{1}} \mathcal{F}$ is a regular sequence, it follows from the theory of filtered rings that there exists an equisingular deformation of $V_{0}$ with general fiber $V_{t}$ (cf. [14, §6], [16]). Hence $V_{t}$ is an isolated complete intersection singularity, and the weighted dual graphs of $V_{0}$ and $V_{t}$ are the same. By Theorem 4.7, the family $\left\{V_{t} \mid t \in \mathbb{C}\right\}$ is an equisingular deformation.

Next assume that $s \geq 2$. Let $\beta: Z \times \mathbb{C} \rightarrow \mathbb{C}^{m} \times \mathbb{C}$ be the trivial family of the weighted blowing up $Z \rightarrow \mathbb{C}^{m}$ with respect to the weight $\mathbf{w}_{1}=\left(w_{1}, \ldots, w_{m}\right)$. The family $\left\{V_{t} \mid t \in \mathbb{C}\right\}$ is naturally embedded in $\mathbb{C}^{m} \times \mathbb{C}$. Let $W_{i}=\mathbb{C}^{m}$ be as above; however we write $x_{i}$ instead of $z_{i}$. Then the cyclic group $\mathcal{C}_{i}$ acts on $W_{i} \times \mathbb{C}$ as in Lemma 4.9. Let $V_{t}^{i} \subset W_{i} \times\{t\}$ be the strict transform of $V_{t}$. Recall that $V_{t} \cap\left\{x_{1}=x_{2}=0\right\}=\{o\}$ by Corollary 4.5 and that the action of the cyclic group $\mathcal{C}_{i}$ on $V_{t}^{i}$ is free outside the exceptional locus. Thus to prove that $V_{t}$ has an isolated singularity at the origin, it suffices to show that any component of singular loci of $V_{t}^{1}$ and $V_{t}^{2}$, intersecting the exceptional set, is an isolated point. Note that the exceptional divisor is singular at singular points of $V_{t}^{i}$. By Theorem 4.7, Lemma 4.8 and 4.9 , it is enough to prove the following three claims (the claims on $V_{t}^{2}$ are proved in the same way).

Claim 1. The family of exceptional divisors $E_{t} \subset V_{t}^{1}$ is trivial.

Claim 2. Let $p \in E_{0}$ be a singular point and let $p_{t} \in E_{t}$ denote the point $p$ under the identification $E_{0}=E_{t}$. Then each $\left(V_{t}^{1}, p_{t}\right)$ is an isolated singularity and the weighted dual graph of $\left(V_{t}^{1}, x_{t}\right)$ is independent of $t$.

Claim 3. If $q_{1}, \ldots, q_{k} \in V_{0}^{1}$ be the fixed points of $\mathcal{C}_{1}$-action, then the fixed locus of the family $\left\{V_{t}^{1} \mid t \in \mathbb{C}\right\}$ is $\bigcup_{j}\left\{q_{j}\right\} \times \mathbb{C}$. If $\mathcal{C}_{1, j} \subset \mathcal{C}_{1}$ denotes the isotropy group of $\left\{q_{j}\right\} \times \mathbb{C}$, then Lemma 4.9 applies to the family $\left\{\left(V_{t}^{1}, q_{j}\right) \mid t \in \mathbb{C}\right\}$ with $\mathcal{C}_{1, j}$-action for every $q_{j}$.

The exceptional divisor $E_{t}$ is defined by $x_{1}=0$ in $V_{t}^{1}$. The ideal of $V_{t}^{1} \subset \mathbb{C}^{m}=$ $W_{1} \times\{t\}$ is generated by the following functions:

$$
\begin{aligned}
F_{11} & =1+a_{11} \mathbf{x}_{d_{1}-1}^{\alpha_{d_{1}-1}}+b_{11} \mathbf{m}_{1 d_{1}}+t \bar{f}_{11}^{+}, & & \\
F_{1 j} & =\mathbf{x}_{j}^{\alpha_{j}}+a_{1 j} \mathbf{x}_{d_{1}-1}^{\alpha_{d_{1}-1}}+b_{1 j} \mathbf{m}_{1 d_{1}}+t \bar{f}_{1 j}^{+}, & & 2 \leq j \leq d_{1}-2, \\
F_{i j} & =\mathbf{m}_{i j}+a_{i j} \mathbf{m}_{i d_{i}-1}+b_{i j} \overline{\mathbf{m}}_{i d_{i}}+t \bar{f}_{i j}^{+}, & & i \geq 2,1 \leq j \leq d_{i}-2,
\end{aligned}
$$

where

$$
\bar{f}_{i j}^{+}=f_{i j}^{+}\left(x_{1}^{w_{1}}, x_{1}^{w_{2}} x_{2}, \ldots, x_{1}^{w_{m}} x_{m}\right) / x_{1}^{\mathbf{w}_{1}-\operatorname{ord}\left(\mathbf{f}_{i j}\right)},
$$

and $\overline{\mathbf{m}}_{i d_{i}}$ is obtained in the same way from $\mathbf{m}_{i d_{i}}$. Recall the condition on the order of $f_{i j}^{+}$, and that

$$
A_{1}-\operatorname{deg}\left(m_{i j}\right)<A_{1}-\operatorname{deg}\left(m_{i d_{i}}\right), \quad i \geq 2,1 \leq j \leq d_{i}-2 .
$$


Then we see that the ideal of $E_{t} \subset \mathbb{C}^{m}$ is generated by $x_{1}$ and the following polynomials:

$$
\begin{array}{ll}
1+a_{11} \mathbf{x}_{d_{1}-1}^{\alpha_{d_{1}-1}}+b_{11} \mathbf{m}_{1 d_{1}}, & \\
\mathbf{x}_{j}^{\alpha_{j}}+a_{1 j} \mathbf{x}_{d_{1}-1}^{\alpha_{d_{1}-1}}+b_{1 j} \mathbf{m}_{1 d_{1}}, & 2 \leq j \leq d_{1}-2, \\
\mathbf{m}_{i j}+a_{i j} \mathbf{m}_{i d_{i}-1}, & i \geq 2,1 \leq j \leq d_{i}-2 .
\end{array}
$$

These polynomials do not contain $t$; thus Claim 1 is verified. Since the fixed points of the $\mathcal{C}_{j}$-action lie on $E_{t}$, the first assertion of Claim 3 follows. By looking at the action explicitly, we see that the $\mathcal{C}_{1, j}$-action on $W_{1} \times \mathbb{C}$ is unitary around $q_{j}$. Thus Claim 3 follows from Claim 2.

It is easy to see that there are $m-d_{1}$ polynomials in (4.5) and any of them contains no variables $x_{1}, \ldots, x_{d_{1}-1}$. As in the proof of Lemma 4.4 , we can show that the functions of (4.5) define a complete intersection curve $C^{\prime} \subset \mathbb{C}^{m-d_{1}+1}$ which is smooth except for the origin. Furthermore (4.3) and (4.4) define a tower of cyclic coverings over $C^{\prime}$. Hence the singularities of $E_{t}$ are lying above the point $(0, \ldots, 0)$ of $C^{\prime}$. Therefore at each singular point of $E_{t}$, the only $d_{1}-2$ variables $x_{2}, \ldots, x_{d_{1}-1}$ are nonzero, and others are zero.

We fix $t \in \mathbb{C}$. Let $p$ be a singular point of $E_{t}$. At $p$, the Jacobian matrix

$$
\partial\left(F_{11}, \ldots, F_{1 d_{1}-2}\right) / \partial\left(x_{2}, \ldots, x_{d_{1}-1}\right)
$$

is regular, and thus $x_{2}, \ldots, x_{d_{1}-1}$ can be expressed by a convergent power series with nonzero constant terms in $x_{1}, x_{d_{1}}, \ldots, x_{m}$. By substituting them into $F_{i j}$, $i \geq 2$, we obtain new defining functions for the germ $\left(V_{t}^{1}, p\right)$ in $\left(\mathbb{C}^{m-d_{1}+2}, o\right)$ as follows:

$$
F_{i j}^{\prime}:=\mathbf{m}_{i j}+a_{i j} \mathbf{m}_{i d_{i}-1}+b_{i j}^{\prime} \overline{\mathbf{m}}_{i d_{i}}^{\prime}+r_{i j}, \quad i \geq 2, \quad 1 \leq j \leq d_{i}-2,
$$

where $\overline{\mathbf{m}}_{i d_{i}}^{\prime}$ is a monomial obtained by substituting 1 into $x_{2}, \ldots, x_{d_{1}-1}$ of $\overline{\mathbf{m}}_{i d_{i}}$. We see that

$$
a_{i j_{1}} b_{i j_{2}}^{\prime} \neq a_{i j_{2}} b_{i j_{1}}^{\prime}\left(j_{1} \neq j_{2}\right) \text { and } \quad b_{i j}^{\prime} \neq 0 .
$$

By (4.1), for every $\mathbb{Q}$-monomial $m=x(D)$, we have

$$
\mathbf{w}_{1}-\operatorname{deg}(\mathbf{m})=e_{1} \cdot A_{1}-\operatorname{deg}(m)=e_{1} \cdot m_{A_{1}}(D) .
$$

Suppose that $m_{i d_{i}}=x\left(D_{i}\right)$ for $i \geq 2$. Since $A_{1}-\operatorname{deg}\left(m_{i d_{i}-1}\right)=m_{A_{1}}\left(\bar{A}_{i}\right)$, the exponent of $x_{1}$ in $\overline{\mathbf{m}}_{i d_{i}}^{\prime}$ is

$$
\mathbf{w}_{1}-\operatorname{deg}\left(\mathbf{m}_{i d_{i}}\right)-\mathbf{w}_{1}-\operatorname{deg}\left(\mathbf{m}_{i d_{i}-1}\right)=e_{1} m_{A_{1}}\left(D_{i}-\bar{A}_{i}\right)>0 .
$$

Since $D_{i}-\bar{A}_{i} \in A_{\mathbb{Z}}$, it follows that $\overline{\mathbf{m}}_{i d_{i}}^{\prime}$ is a monomial of $x_{1}^{e_{1}}, \mathbf{x}_{d_{1}}, \ldots, \mathbf{x}_{m}$. We denote by $h_{i}$ the monomial obtained by replacing $x_{1}^{e_{1}}, \mathbf{x}_{d_{1}}, \ldots, \mathbf{x}_{m}$ of $\overline{\mathbf{m}}_{i d_{i}}^{\prime}$ with $x_{1}, x_{d_{1}}, \cdots, x_{m}$, respectively. Let $\delta^{\prime}=\left(e_{1}, \delta_{d_{1}}, \ldots, \delta_{m}\right) \in \mathbb{N}^{m-d_{1}+2}$ and let

$$
f_{i j}^{\prime}=m_{i j}+a_{i j} m_{i d_{i}-1}+b_{i j}^{\prime} h_{i}, \quad i \geq 2, \quad 1 \leq j \leq d_{i}-2 .
$$

Then the polynomial $\mathbf{m}_{i j}+a_{i j} \mathbf{m}_{i d_{i}-1}+b_{i j}^{\prime} \overline{\mathbf{m}}_{i d_{i}}^{\prime}$ is the $\delta^{\prime}$-lifting of $f_{i j}^{\prime}$.

Let $A_{e}$ be the component of the branch $C_{d_{1}}$ of the node $A_{1}$, which intersects $A_{1}$; see Figure 3. We may assume that the $A_{e}$ is an end of $C_{d_{1}}$; take the blowing up at $A_{1} \cap A_{e}$ if necessary. Let $A^{\prime}=C_{d_{1}}$. Then $A^{\prime} \subset M$ can be blown down to a normal surface singularity. We consider admissible forms concerning $A^{\prime}$. We associate the end $A_{e}$ with the variable $x_{1}$, while keeping the correspondence between the other ends and the variables $x_{d_{1}}, \ldots, x_{m}$. 


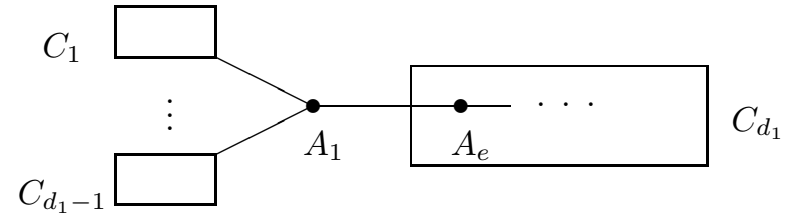

FiguRe 3.

Claim 4. We have the following.

(1) $A^{\prime}$ has $s-1$ nodes $A_{2}, \cdots, A_{s}$.

(2) $A^{\prime}$ satisfies Condition 3.3, and for each $2 \leq i \leq s$, the set of polynomials

$$
\left\{f_{i 1}^{\prime}, \ldots, f_{i d_{i}-2}^{\prime}\right\}
$$

is a Neumann-Wahl system concerning $A^{\prime}$ at a node $A_{i}$.

(3) In this new situation, we define a weight $\mathbf{w}_{i}^{\prime} \in \mathbb{N}^{m-d_{1}+2}(2 \leq i \leq s)$ with respect to $\delta^{\prime}$ as in the sentence before Definition 4.1. Then

$$
\mathbf{w}_{i}^{\prime} \text {-deg }\left(\mathbf{m}_{i j}+a_{i j} \mathbf{m}_{i d_{i}-1}+b_{i j}^{\prime} \overline{\mathbf{m}}_{i d_{i}}^{\prime}\right)<\mathbf{w}_{i}^{\prime} \text {-ord }\left(r_{i j}\right) .
$$

The inductive hypothesis and Claim 4 imply that $\left(V_{t}^{1}, p\right)$ is an isolated singularity and is an equisingular deformation of the singularity defined by the $\delta^{\prime}$-lifting of the Neumann-Wahl system $\left\{f_{i j}^{\prime}\right\}$; thus Claim 2 follows.

Now we prove Claim 4. First, the assertion (1) is obvious. It is clear that the number of branches of a node $A_{i}(i \geq 2)$ in $A^{\prime}$ is the same as that of $A_{i}$ in $A$. For $D=\sum a_{j} A_{j} \in A_{\mathbb{Q}}$, we write $\operatorname{res}(D)=\sum_{A_{j} \leq A^{\prime}} a_{j} A_{j}$. Let $\widetilde{A}_{k}$ denote the dual cycle of $A_{k}$ on $A^{\prime}$. Let $D_{i j}(i \geq 2)$ be the monomial cycle such that $x\left(D_{i j}\right)=m_{i j}$, and let

$$
E_{i j}=\operatorname{res}\left(D_{i j}-\bar{A}_{i}\right)
$$

Since $\left(D_{i j}-\bar{A}_{i}\right) \cdot A_{e}=0$, we have $-E_{i j} \cdot A_{e}=m_{A_{1}}\left(D_{i j}-\bar{A}_{i}\right)$; this is the exponent of $x_{1}$ in $h_{i}$ (see (4.8)). By computing the intersection numbers $\left(E_{i j}+\widetilde{A}_{i}\right) \cdot A_{l}$ for every $A_{l} \leq A^{\prime}$, we see that $E_{i j}+\widetilde{A}_{i}$ is a monomial cycle belonging to a branch of $A_{i}$ and that

$$
x\left(E_{i d_{i}}+\widetilde{A}_{i}\right)=h_{i} \quad \text { and } \quad x\left(E_{i j}+\widetilde{A}_{i}\right)=m_{i j} \quad \text { for } 1 \leq j<d_{i} .
$$

By (4.7), we have (2) of Claim 4. We can generalize the argument above as follows. Let $m$ be any monomial in $\mathbf{f}_{i j}+t f_{i j}^{+}$. Suppose that $m$ is the $\delta$-lifting of a $\mathbb{Q}$-monomial $x(D)$ associated with a $\mathbb{Q}$-monomial cycle $D \in A_{\mathbb{Q}}$. Let $m^{\prime}$ be the $\delta^{\prime}$-lifting of the monomial $x\left(\operatorname{res}\left(D-\bar{A}_{i}\right)+\widetilde{A}_{i}\right)$. Then, by the operation which changes $\mathbf{f}_{i j}+t f_{i j}^{+}$ into the function $F_{i j}^{\prime}$, the monomial $m$ is changed into a function of the form $u m^{\prime}$, where $u \in S$ is a unit. We have that $m_{A_{i}}\left(\operatorname{res}\left(D-\bar{A}_{i}\right)+\widetilde{A}_{i}\right)=m_{A_{i}}(D)$ for a node $A_{i}(i \geq 2)$. Now it is easy to see that (3) holds. Thus we have proved Claim 4.

Let $\hat{S}=\mathbb{C}\left[\left[x_{1}, \ldots, x_{m}\right]\right]$ denote the formal power series ring. By a similar argument as in the proof of Theorem 4.3, we obtain the following.

Theorem 4.10. Let $\mathbf{f}_{i j}$ be as above. Take a formal power series $g_{i j} \in \hat{S}$ such that

$$
\mathbf{w}_{i}-\operatorname{deg}\left(\mathbf{f}_{i j}\right)<\mathbf{w}_{i} \operatorname{ord}\left(g_{i j}\right) \quad \text { for } \quad 1 \leq i \leq s, 1 \leq j \leq d_{i}-2 .
$$

Let $J \subset \hat{S}$ be the ideal generated by all $\mathbf{f}_{i j}+g_{i j}$ 's. Then $\hat{S} / J$ is a two-dimensional complete intersection ring, and has only a singularity at the maximal ideal. 


\section{The MAIN RESUlts}

In this section we will prove the following.

Theorem 5.1. If $(X, o)$ is a rational or minimally elliptic singularity, then its universal abelian cover $(Y, o)$ is an equisingular deformation of a Neumann-Wahl complete intersection singularity. The deformation is defined by the functions of the form $f+t f^{+}$, where $\{f\}$ is a Neumann-Wahl system associated with the exceptional set $A, f^{+}$is a function with order greater than the degree of $f$ and $t$ is the parameter.

We start without the assumption of the theorem. We use the notation of Section 2. Write $\mathcal{A}_{b}=H^{0}\left(-L^{(b)}\right)$ and $\mathcal{O}_{Y, o}=\bigoplus_{b \in \mathcal{B}} \mathcal{A}_{b}$. Recall that for any component $A_{i}$ of $A$, there exist a divisor $L^{i}$ and an element $b^{i} \in \mathcal{B}$ such that $\nu\left(L^{i}\right)=\bar{A}_{i}$ and $L^{i}-L^{\left(b^{i}\right)} \in A_{\mathbb{Z}}$. We consider the following condition, which depends on the resolution $\pi: M \rightarrow X$.

Condition 5.2. For each end $A_{i} \in \mathcal{E}(A)$, there exists a section $y_{i} \in H^{0}\left(-L^{i}\right)$ such that $\left(y_{i}\right)_{A}=\nu\left(L^{i}\right)$.

Lemma 5.3. If $(X, o)$ is rational, or if $(X, o)$ is minimally elliptic and the minimally elliptic cycle $E$ is supported on A, then Condition 5.2 is satisfied.

Proof. Let $D$ be any $\pi$-nef divisor on $M$. If $(X, o)$ is rational, then $D$ is $\pi$-free; see $[13,4.17]$. Assume that $(X, o)$ is minimally elliptic and the minimally elliptic cycle $E$ is supported on $A$. Since $E$ is 2-connected, $H^{0}(D)$ has no fixed component on $A$ if $D \cdot A \geq 1$ by $[13,4.23$, Remark]. Thus the assertion follows.

Let $\mathfrak{m}_{Y}$ (resp. $\mathfrak{m}_{X}$ ) denote the maximal ideal of $\mathcal{O}_{Y, o}$ (resp. $\mathcal{O}_{X, o}$ ). We may identify $\mathfrak{m}_{Y}$ as $\mathfrak{m}_{X} \bigoplus\left(\bigoplus_{b \neq 0} \mathcal{A}_{b}\right)($ cf. $[12, \S 6])$.

Lemma 5.4. Let $\left\{Z_{k} \in A_{\mathbb{Z}} \mid k \in \mathbb{N}\right\}$ be a sequence of cycles such that $Z_{k+1}>Z_{k}>0$ for every $k \in \mathbb{N}$. Then there exists a function $\alpha: \mathbb{N} \rightarrow \mathbb{N}$ such that for each $b \in \mathcal{B}$,

$$
H^{0}\left(-L^{(b)}-Z_{k}\right) \subset \mathfrak{m}_{Y}^{\alpha(k)} \text { and } \lim _{k \rightarrow \infty} \alpha(k)=\infty .
$$

Proof. We only give an outline. We can take a positive integer $a$ so that for any $\pi$-nef divisor $D$ on $M$ and a cycle $Z:=a \sum_{A_{i} \leq A} \bar{A}_{i}$, the natural map

$$
H^{0}(D-Z) \otimes H^{0}(-Z) \rightarrow H^{0}(D-2 Z)
$$

is surjective (cf. $[5, \mathrm{III}]$ ). Let $\beta$ be a nonnegative integer such that $-L^{(b)}-\beta Z$ is $\pi$-nef for every $b \in \mathcal{B}$. Then we obtain $H^{0}\left(-L^{(b)}-(\beta+k) Z\right) \subset \mathfrak{m}_{Y}^{k}$. We may assume $Z_{1}>(\beta+1) Z$. Now define $\alpha(l)=\max \left\{k \in \mathbb{N} \mid(\beta+k) Z \leq Z_{l}\right\}$.

Assumption 5.5. From now on, we assume that Condition 3.4 and 5.2 are satisfied.

If $A$ is a chain of curves, then $(X, o)$ is a cyclic quotient singularity and $\mathcal{O}_{Y, o}=$ $\mathbb{C}\left\{y_{1}, y_{2}\right\}$, where $y_{i}$ 's are as in Condition 5.2 (if $A$ is irreducible, then $y_{1}, y_{2} \in$ $\left.H^{0}\left(-L^{1}\right)\right)$. Let $m=\# \mathcal{E}(A)$. Assume that $m \geq 3$. Then we can define admissible monomials at each node by associating each end with a variable as in Section 3 . We define the homomorphism

$$
\psi: S=\mathbb{C}\left\{x_{1}, \ldots, x_{m}\right\} \rightarrow \mathcal{A}=\mathcal{O}_{Y, o}
$$

of $\mathbb{C}$-algebras by $\psi\left(x_{i}\right)=y_{i}$. We denote by ${ }^{\wedge}$ the maximal-ideal-adic completions of local rings. Let $\hat{\psi}: \hat{S}=\mathbb{C}\left[\left[x_{1}, \ldots, x_{m}\right]\right] \rightarrow \hat{\mathcal{O}}_{Y, o}$ be the induced homomorphism. By 
the definition of the set $\mathcal{B}$, we may regard $\mathcal{B}$ as the discriminant group $G:=\bar{A}_{\mathbb{Z}} / A_{\mathbb{Z}}$ in the natural way. Let $S_{b} \subset S$ (resp. $\left.\hat{S}_{b} \subset \hat{S}\right), b \in \mathcal{B}$, denote the set of power series represented as the sum of monomials $x(D)$ satisfying $D\left(\bmod A_{\mathbb{Z}}\right)=b$. Then we have $S=\bigoplus_{b \in \mathcal{B}} S_{b}$ and the $\psi$ becomes a homomorphism of $\mathcal{B}$-graded (or $G$-graded) algebras. The same holds for $\hat{S}$ and $\hat{\psi}$.

Let $A_{1}, \ldots, A_{s}$ be all of the nodes of $A$, and $d_{i}$ the number of branches of a node $A_{i}$. Let $\mathcal{M}_{i}=\left\{m_{i 1}, \ldots, m_{i d_{i}}\right\}$ denote a complete system of admissible monomials at a node $A_{i}$. Let $\mathbb{C} \mathcal{M}_{i} \subset S$ denote the $\mathbb{C}$-linear subspace spanned by the monomials of $\mathcal{M}_{i}$.

Lemma 5.6. For any node $A_{i}$, let $\mu_{i}$ denote the composite of homomorphisms

$$
\mathbb{C} \mathcal{M}_{i} \stackrel{\psi}{\longrightarrow} H^{0}\left(\mathcal{O}_{M}\left(-L^{i}\right)\right) \rightarrow H^{0}\left(\mathcal{O}_{A_{i}}\left(-L^{i}\right)\right) .
$$

Then $\mu_{i}$ is surjective. We have $h^{0}\left(\mathcal{O}_{A_{i}}\left(-L^{i}\right)\right)=2$ and $\operatorname{dim} \operatorname{Ker} \mu_{i}=d_{i}-2$. Let $\mathcal{F}_{i}=\left\{f_{i 1}, \ldots, f_{i d_{i}-2}\right\}$ be a basis of Ker $\mu_{i}$. Then $\mathcal{F}_{i}$ is a Neumann-Wahl system at the node $A_{i}$.

Proof. Since $L^{i} \cdot A_{i}=-1$, we have $h^{0}\left(\mathcal{O}_{A_{i}}\left(-L^{i}\right)\right)=2$. Suppose $m_{i j}=x\left(D_{i j}\right)$ for a monomial cycle $D_{i j}$ belonging to a branch $C_{i j}$ of $A_{i}$. Since $\left(D_{i j}-\bar{A}_{i}\right) \cdot A_{i}=1$, it follows from Lemma 2.1 that $\mu_{i}\left(x\left(D_{i j}\right)\right)$ has a zero of order one at $A_{i} \cap C_{i j}$. Thus $\mu_{i}\left(x\left(D_{i d_{i}-1}\right)\right)$ and $\mu_{i}\left(x\left(D_{i d_{i}}\right)\right)$ generate $H^{0}\left(\mathcal{O}_{A_{i}}\left(-L^{i}\right)\right)$. Therefore we obtain a complete system of admissible forms expressed by a $((p-2) \times p)$-matrix as in Remark 3.7, which is a basis of Ker $\mu_{i}$.

Let us recall that polynomials of $\mathcal{F}_{i}$ are quasihomogeneous with respect to the $A_{i}$-weight.

Lemma 5.7. Let $A_{i}$ be a node and $h \in H^{0}\left(-L^{i}\right)$. Then there exists $\bar{h} \in \hat{S}_{b^{i}}$ such that $\hat{\psi}(\bar{h})=h$ in $\hat{\mathcal{O}}_{Y, o}$. Suppose that $h=\psi\left(\bar{h}_{0}\right)$ for an admissible form $\bar{h}_{0} \in \operatorname{Ker} \mu_{i}$. Then we can take the $\bar{h}$ so that $A_{i}-\operatorname{deg}\left(\bar{h}_{0}\right)<A_{i}-\operatorname{ord}(\bar{h})$. If in addition $\psi$ is surjective, such $\bar{h}$ can be taken from $S_{b^{i}}$.

Proof. We use the notation of Lemma 5.6. Suppose that $h \neq 0$. Let $F_{0}$ be the divisor such that $\nu\left(F_{0}\right)=(h)_{A}$ and $F_{0}-L^{i} \in A_{\mathbb{Z}}$. Let $c_{0}=-F_{0} \cdot A_{i}$. Then $c_{0} \geq 0$. By Condition 3.4 and the proof of Lemma 3.5, there exists a cycle $F_{0}^{\prime} \geq F_{0}$ such that $m_{A_{i}}\left(F_{0}^{\prime}-F_{0}\right)=0,\left(F_{0}^{\prime}-F_{0}\right) \cdot A_{i}=0$ and $F_{0}^{\prime} \cdot A_{j}=0$ if $j \neq i$ and $A_{j}$ is not an end. Let $D_{i j}^{\prime}=D_{i j}-\bar{A}_{i}$. Then an arbitrary cycle of the form $E_{\mathbf{a}}:=F_{0}^{\prime}+\sum a_{j} D_{i j}^{\prime}$ is a monomial cycle, where $\mathbf{a}=\left(a_{1}, \ldots, a_{m}\right) \in \mathbb{Z}^{m}$ with $\sum a_{j}=c_{0}$ and $a_{j} \geq 0$. It is easy to see that the $\mu_{i}\left(x\left(E_{\mathbf{a}}\right)\right)$ 's span $H^{0}\left(\mathcal{O}_{A_{i}}\left(-F_{0}\right)\right)$. Thus we have a quasihomogeneous polynomial $\bar{h}_{1}$, which is a linear form of $x\left(E_{\mathbf{a}}\right)$ 's, with respect to $A_{i}$-weight such that $h-\psi\left(\bar{h}_{1}\right) \in H^{0}\left(-F_{0}-A_{i}\right)$. Let $F_{1}$ be the divisor such that $\nu\left(F_{1}\right)=\left(h-\psi\left(\bar{h}_{1}\right)\right)_{A}$ and $F_{1}-L^{i} \in A_{\mathbb{Z}}$. Then it follows from the argument above that there exists a quasihomogeneous polynomial $\bar{h}_{2}$ such that

$$
h-\psi\left(\bar{h}_{1}\right)-\psi\left(\bar{h}_{2}\right) \in H^{0}\left(-F_{1}-A_{i}\right) .
$$

Thus we obtain a sequence $\left\{\bar{h}_{k} \mid k \in \mathbb{N}\right\}$ of quasihomogeneous polynomials and a sequence $\left\{F_{k} \mid k \in \mathbb{N}\right\}$ of divisors satisfying the following: for all $k \in \mathbb{N}$,

(1) $h-\psi\left(\bar{h}_{1}+\cdots+\bar{h}_{k}\right) \in H^{0}\left(-F_{k}\right)$,

(2) $F_{k+1}>F_{k}$

(3) $A_{i}-\operatorname{deg}\left(\bar{h}_{k}\right)<A_{i}-\operatorname{deg}\left(\bar{h}_{k+1}\right)$. 
By Lemma 5.4 there exists a function $\alpha: \mathbb{N} \rightarrow \mathbb{N}$ such that $H^{0}\left(-F_{k}\right) \subset \mathfrak{m}_{Y}^{\alpha(k)}$ and that $\lim _{k \rightarrow \infty} \alpha(k)=\infty$. Now put $\bar{h}=\sum \bar{h}_{i} \in \hat{S}_{b^{i}}$. Then $\hat{\psi}(\bar{h})=h$. Suppose that $h=\psi\left(\bar{h}_{0}\right)$ with an admissible form $\bar{h}_{0} \in \operatorname{Ker} \mu_{i}$. Since $\psi\left(\bar{h}_{0}\right) \in H^{0}\left(-L^{i}-A_{i}\right)$, we have

$$
A_{i}-\operatorname{deg}\left(\bar{h}_{0}\right)<m_{A_{i}}\left(F_{0}\right)=A_{i}-\operatorname{deg}\left(\bar{h}_{1}\right) .
$$

If $\psi$ is surjective, then the maps $\left(x_{1}, \cdots, x_{m}\right)^{k} \rightarrow \mathfrak{m}_{Y}^{k}$ and $S_{b} \rightarrow \mathcal{A}_{b}$ are surjective for every $k \in \mathbb{N}$ and $b \in \mathcal{B}$. Therefore, for sufficiently large $k$, there exists $\bar{h}^{\prime} \in S_{b^{i}}$ such that $A_{i}-\operatorname{ord}\left(\bar{h}^{\prime}\right)>A_{i}-\operatorname{deg}\left(\bar{h}_{0}\right)$ and $h=\psi\left(\bar{h}_{1}+\cdots+\bar{h}_{k}+\bar{h}^{\prime}\right)$.

Proposition 5.8. The homomorphism $\psi: S \rightarrow \mathcal{O}_{Y, o}$ is surjective.

Proof. We fix a node $A_{i}$. By [11, Proposition 5.1], the group $G=\bar{A}_{\mathbb{Z}} / A_{\mathbb{Z}}$ is generated by $\left\{\bar{A}_{j} \mid A_{j} \in \mathcal{E}(A)\right\}$. Hence for each $b \in \mathcal{B}$, there exists a monomial $m_{b} \in S$ such that $\mathcal{A}_{b} \cdot m_{b} \subset H^{0}\left(-L^{i}\right)$. By Lemma 5.7, we have $\mathcal{A}_{b} \subset \hat{\psi}\left(\hat{S}_{b}\right) \cdot m_{b}^{-1}$. Therefore

$$
\hat{\psi}(\hat{S}) \subset \hat{\mathcal{O}}_{Y, o} \subset \sum_{b \in \mathcal{B}} \hat{\psi}(\hat{S}) \cdot m_{b}^{-1} .
$$

Then it follows that $\hat{S} / \operatorname{Ker} \hat{\psi}$ is a two-dimensional domain. By Lemma 5.7, for any $f_{k l} \in \bigcup \mathcal{F}_{j}$, there exists $\tilde{f}_{k l} \in \hat{S}$ such that $\operatorname{LF}_{A_{k}}\left(\tilde{f}_{k l}\right)=f_{k l}$ and $\tilde{f}_{k l} \in \operatorname{Ker} \hat{\psi}$. Let $\tilde{I} \subset \hat{S}$ denote the ideal generated by all $\tilde{f}_{k l}$ 's. Then it follows from Theorem 4.10 that $\hat{S} / \tilde{I}$ is a two-dimensional normal domain. Since $\tilde{I} \subset \operatorname{Ker} \hat{\psi}$, we obtain that $\hat{S} / \tilde{I} \cong \hat{\psi}(\hat{S})$. Since $\hat{S}$ is Noetherian, $\hat{\mathcal{O}}_{Y, o}$ is finitely generated $\hat{\psi}(\hat{S})$-module. By the normality of $\hat{\psi}(\hat{S})$, we obtain $\hat{\psi}(\hat{S})=\hat{\mathcal{O}}_{Y, o}$. This implies that $\psi$ is surjective.

It follows from Lemma 5.7 and Proposition 5.8 that for each $f_{i j} \in \mathcal{F}_{i}$, there exists $f_{i j}^{+} \in S_{b^{i}}$ such that $f_{i j}+f_{i j}^{+} \in \operatorname{Ker} \psi$ and $A_{i}$-deg $\left(f_{i j}\right)<A_{i}$-ord $\left(f_{i j}^{+}\right)$. For each $t \in \mathbb{C}$, we denote by $I_{t} \subset S$ the ideal generated by the functions

$$
f_{i j}+t f_{i j}^{+}, \quad 1 \leq i \leq s, 1 \leq j \leq d_{i}-2 .
$$

As in the proof of Proposition 5.8, by Theorem 4.3, we see that $\operatorname{Ker} \psi=I_{1}$.

Corollary 5.9. $\mathcal{O}_{Y, o} \cong S / I_{1}$.

Again by Theorem 4.3, we obtain the following.

Theorem 5.10. If Condition 3.4 and 5.2 are satisfied, then the universal abelian cover $(Y, o)$ of $(X, o)$ is an equisingular deformation of a Neumann-Wahl complete intersection singularity. The deformation is defined by the functions

$$
f_{i j}+T f_{i j}^{+} \in S[T], \quad 1 \leq i \leq s, 1 \leq j \leq d_{i}-2,
$$

where $S[T]$ is the polynomial ring over $S$.

Now Theorem 5.1 follows from Theorem 5.10, Lemma 3.5 and 5.3.

Corollary 5.11. If Condition 3.4 and 5.2 are satisfied, then $(X, o)$ is $\mathbb{Q}$-Gorenstein. If in addition the link of $(X, o)$ is a homology sphere, then $(X, o)$ is an equisingular deformation of a Neumann-Wahl complete intersection singularity.

Remark 5.12. There is ambiguity in the choice of the complete systems of admissible monomials $\mathcal{M}_{i}$. The proof of Lemma 5.7 shows that if $m$ and $m^{\prime}$ belong to the same branch of a node $A_{i}$, then there exists $h \in S_{b^{i}}$ such that $x(m)-x\left(m^{\prime}\right)-h \in \operatorname{Ker} \psi$ and $A_{i}$-ord $(h)>A_{i}-\operatorname{deg}(x(m))$. Therefore, whether $(Y, o)$ is a Neumann-Wahl complete intersection depends not only on the choice of the sections $\left\{y_{i}\right\}$, but also on the choice of the complete systems of admissible monomials. 
In the rest of this section, we describe the action of the Galois group of the universal abelian covering. Recall that the Galois group $H_{1}(\Sigma, \mathbb{Z})$ of the universal abelian covering $(Y, o) \rightarrow(X, o)$ is isomorphic to the discriminant group $G=\bar{A}_{\mathbb{Z}} / A_{\mathbb{Z}}$.

For any $\mathbb{Q}$-cycle $D=\sum a_{i} \bar{A}_{i}=\sum b_{i} A_{i}$, we have $D \cdot A_{j}=-a_{j}$ and $D \cdot \bar{A}_{j}=-b_{j}$. Thus we obtain the following

Lemma 5.13. Let $D \in A_{\mathbb{Q}}$. Then

(1) $D \in \bar{A}_{\mathbb{Z}}$ if and only if $\left\{D \cdot A_{i} \mid A_{i} \leq A\right\} \subset \mathbb{Z}$,

(2) $D \in A_{\mathbb{Z}}$ if and only if $\left\{D \cdot \bar{A}_{i} \mid A_{i} \leq A\right\} \subset \mathbb{Z}$.

For any $D \in \bar{A}_{\mathbb{Z}}$, we denote by $(D)$ the class of $D$ modulo $A_{\mathbb{Z}}$. Then the action of $G$ on $S=\mathbb{C}\left\{x_{1}, \ldots, x_{m}\right\}$ is expressed as follows (cf. [11, $\left.\S 5\right]$ ). For $(D) \in G$ and a monomial cycle $F$,

$$
(D) \cdot x(F):=\exp (2 \pi \sqrt{-1} D \cdot F) x(F) .
$$

By Lemma $5.13(1)$, this is well-defined. Let $\left(Y_{t}, o\right)$ denote the singularity defined by the ideal $I_{t} \subset S$. We have seen that $\left\{Y_{t} \mid t \in \mathbb{C}\right\}$ is an equisingular family. Since $I_{t}$ is generated by homogeneous elements of $G$-graded algebra $S$, the group $G$ naturally acts on $S / I_{t}=\mathcal{O}_{Y_{t}, o}$. It follows from Lemma 5.13 (2) that $\mathcal{O}_{X, o}=\left(\mathcal{O}_{Y, o}\right)^{G}$. By Corollary 4.5 and [11, Proposition 5.2], the action is free on $Y_{t} \backslash\{o\}$ (see also [11, Theorem $7.2(2)])$. Therefore $X_{t}:=Y_{t} / G$ is a normal singularity. The linear action of $G$ on $\mathbb{C}^{m}$ determined by (5.1) is unitary. By Lemma 4.9 and the uniqueness of the universal abelian covering, we obtain the following

Theorem 5.14. The family $\left\{X_{t} \mid t \in \mathbb{C}\right\}$ is an equisingular deformation, and each $Y_{t} \rightarrow X_{t}$ is the universal abelian covering.

\section{REFERENCES}

[1] E. Brieskorn, Singular elements of semi-simple algebraic groups, Actes du Congrès International des Mathématiciens (Nice, 1970), Tome 2, Gauthier-Villars, Paris, 1971, pp. 279-284.

[2] S. Ishii, Simultaneous canonical models of deformations of isolated singularities, Algebraic geometry and analytic geometry (Tokyo, 1990), ICM-90 Satell. Conf. Proc., Springer, Tokyo, 1991, pp. 81-100.

[3] H. Laufer, On rational singularities, Amer. J. Math. 94 (1972), 597-608.

[4] H. Laufer, On minimally elliptic singularities, Amer. J. Math. 99 (1977), 1257-1295.

[5] H. Laufer, Weak simultaneous resolution for deformations of Gorenstein surface singularities, Singularities, Part 2 (Arcata, Calif., 1981), Proc. Sympos. Pure Math., vol. 40, Amer. Math. Soc., Providence, R.I., 1983, pp. 1-29.

[6] W. D. Neumann, A calculus for plumbing applied to the topology of complex surface singularities and degenerating complex curves, Trans. Amer. Math. Soc. 268 (1981), 299-344.

[7] W. D. Neumann, Abelian covers of quasihomogeneous surface singularities, Singularities, Part 2 (Arcata, Calif., 1981), Proc. Sympos. Pure Math., vol. 40, Amer. Math. Soc., Providence, RI, 1983, pp. 233-243.

[8] W. D. Neumann and J. Wahl, Universal abelian covers of surface singularities, Trends in singularities, Trends Math., Birkhäuser, Basel, 2002, pp. 181-190.

[9] W. D. Neumann and J. Wahl, Complex surface singularities with integral homology sphere links, 2003, preprint (math.AG/0301165).

[10] W. D. Neumann and J. Wahl, Universal abelian covers of quotient-cusps, Math. Ann. 326 (2003), 75-93.

[11] W. D. Neumann and J. Wahl, Complete intersection singularities of splice type as universal abelian covers, 2004, preprint (math.AG/0407287).

[12] T. Okuma, Universal abelian covers of rational surface singularities, J. London Math. Soc. (2) 70 (2004), 307-324.

[13] M. Reid, Chapters on algebraic surfaces, Complex algebraic geometry, IAS/Park City Math. Ser., vol. 3, Amer. Math. Soc., Providence, RI, 1997, pp. 3-159. 
[14] M. Tomari and K.-i. Watanabe, Filtered rings, filtered blowing-ups and normal twodimensional singularities with "star-shaped" resolution, Publ. Res. Inst. Math. Sci. 25 (1989), $681-740$.

[15] J. Wahl, Equisingular deformations of normal surface singularities, I, Ann. of Math. 104 (1976), 325-365.

[16] J. Wahl, Deformations of quasi-homogeneous surface singularities, Math. Ann. 280 (1988), $105-128$.

Department of Education, Yamagata University, 1-4-12 Kojirakawa-machi, Yamagata 990-8560, JAPAN

E-mail address: okuma@vc.e.yamagata-u.ac.jp 